\title{
Sondeo de opinión ciudadana a los usuarios de transporte público en el cantón Ambato
}

\author{
Fernando Mayorga-Núñez ${ }^{1}$, Carolina Freire- Paredes², Tatiana Vayas- Carrillo³ \\ Fecha de recepción: 13 de Marzo 2018 \\ Fecha de aceptación: 29 de Marzo 2018
}

\section{Resumen}

En esta investigación se analiza la percepción que tiene la ciudadanía sobre el servicio de transporte urbano de buses de la ciudad de Ambato estudiando aspectos como: Infraestructura de unidades, frecuencia de buses, horarios de servicio, velocidad, paradas, seguridad, comodidad, costo del pasaje y calidad de servicio; para lo cual se aplicaron 802 encuestas a los pasajeros de las 22 líneas de transporte registradas en el Gobierno Autónomo Descentralizado Municipalidad de Ambato - GADMA. Los resultados evidencian que la calidad del servicio se encuentra entre regular y buena, ya que tiene un índice de percepción que bordea los 50 puntos. Los datos obtenidos brindan una visión general del sistema de transporte público, informando a instituciones y autoridades la percepción de la ciudadanía sobre este servicio y puedan tomar decisiones en beneficio de los usuarios.

Palabras clave: Transporte público, percepción, servicio, índice.

\section{Abstract}

This research analyzes citizen's perception about urban bus transportation service in Ambato through the study of aspects like: unit infrastructure, time, speed, bus stops, security, comfort, bus ticket cost and quality service; for which 802 surveys were applied to bus passengers in 22 transportation lines registered in the Autonomous Decentralized Municipality Government of Ambato. The results show that the quality service is considered between regular and good because its perception index is around 50 points. The data provides a general vision of the public transportation service, reporting the citizen's perception about this service to institutions and authorities, so they can make decisions in benefit of the users.

Keywords: Public transportation, perception, service, index.

\section{Introducción}

Los sistemas de transporte son importantes para el funcionamiento de las ciudades ya que movilizan a gran parte de la población a distintos lugares para el desarrollo de sus actividades cotidianas, lo que contribuye a la dinámica social y al crecimiento económico. (Oviedo, Sacanambuy, Matabanchoy y Zambrano, 2016)

A nivel mundial, los sistemas de transporte acompañan al desarrollo de las urbes, sin embargo, en América Latina, el desmesurado crecimiento poblacional junto con la desorganizada expansión de las ciudades y el presupuesto asignado para mantenimiento y desarrollo de infraestructura, ha ocasionado que los sistemas vayan quedando rezagados y presenten problemas para la mayoría de la población que utiliza el transporte público (deficiencias de oferta, mala calidad del servicio y altas tarifas) en detrimento de su calidad de vida. (Banco de Desarrollo de América Latina - CAF, 2011)

En busca de mejorar la calidad de vida de los ciudadanos que utilizan medios de transporte público hoy en día las políticas de movilidad urbana son fundamentales para un adecuado manejo de la congestión vehicular e impactos ambientales, asegurando el bienestar de conductor y peatones. (Sociedad Chilena de Ingeniería de Transporte, 2013).

Vasconcellos y Mendonça (2016) indican que la mayor cantidad de viajes en Transporte Público Colectivo - TPC en las ciudades de América Latina se realizan en autobuses, seguido por microbuses y el metro, siendo las ciudades más representativas Ciudad de México, Santiago y Lima; además la movilidad promedio se ubica en dos viajes al día por habitante. Según datos del Instituto Nacional de Estadística y Censos - INEC (2016), Ecuador posee 1'488.802 vehículos de transporte de pasajeros, donde 23.436 son autobuses $(1,6 \%)$, de los cuales Tungurahua abarca el 6,8\%. Rivera, Mayorga, Vayas y Freire (2017) mencionan que en Ambato existen 392 buses de servicio urbano que representan el $24,8 \%$ del total de buses registrados en Tungurahua; además, tomando en cuenta que en Ambato existe una población de 378.523 personas según el INEC (2018), se calcula una tasa de 10,4 buses de servicio urbano por cada diez mil habitantes.

Uno de los proyectos en marcha para mejorar el servicio de transporte urbano de buses en Ambato es el establecimiento de la caja común, el cual consiste en la unificación de la recaudación diaria de cada cooperativa (Jerpazsol, Los Libertadores, Tungurahua, Unión Ambateña y Vía Flores), y el posterior reparto equilibrado entre todos los socios de las operadoras. Para concretar este proyecto, se han venido instalando mecanismos requeridos como son: contador de pasajeros, rastreo GPS, videovigilancia, habilitación de salidas por las puertas medias y traseras, y la adquisición de modernas unidades. (El Telégrafo, 2017) EI GADMA ha establecido 25 paradas inteligentes en diferentes sectores de la ciudad, con servicios de internet inalámbrico gratuito, tomas de corriente para carga de celulares, tablets o laptops, datos turísticos de la ciudad, pantallas leds en las que se informan líneas y paradas de servicio de transporte urbano, entre otros servicios. Además, las rutas de transporte fueron reajustadas por la Dirección Municipal de Tránsito y se ha colocado nueva nomenclatura en los buses, identificada con número y color. (El Telégrafo, 2017)

Bajo este contexto, es importante analizar la percepción de la ciudadanía de Ambato respecto al sistema de transporte urbano de buses, siendo un aporte para el fortalecimiento de políticas públicas en esta área y, además, el planteamiento de nuevas metas a ser alcanzadas en el futuro en beneficio del TPC, coadyuvando al mejoramiento de la calidad de vida de la población ambateña.

\section{Metodología}

El presente estudio fue realizado a través de investigación científica exploratoria que permite dar una visión general de la percepción de la ciudadanía sobre el servicio de transporte urbano de buses en la ciudad de Ambato. El método utilizado para la recolección de información fue la aplicación de encuestas en las que participaron cuatro encuestadores asignados aleatoriamente a las diferentes rutas de transporte por un periodo de 8 días laborables en horarios de la mañana y tarde, excluyendo las horas pico de circulación vehicular; los datos obtenidos son de carácter cuantitativo y cualitativo.

Al no tener una población definida de usuarios de buses, y tomando como referencia el estudio realizado por Heredia (2015), se aplicaron 802 encuestas a los pasajeros, dentro de las unidades, en cada una de las 22 líneas de transporte urbano registradas en el GADMA con el $95 \%$ de confianza y $5 \%$ de error. Además, para determinar la confiabilidad de la encuesta se aplicó el alfa de Cronbach con un resultado de 0,70 estando dentro del intervalo de aceptación. (George y Mallery, 2003, p. 231)

Las 22 rutas se distribuyen en cinco cooperativas como se muestra en la tabla 1: 
Tabla 1. Cooperativas de transporte público de Ambato

\begin{tabular}{lc}
\hline \multicolumn{1}{c}{ Cooperativa } & $\begin{array}{c}\text { Nro. Rutas asignadas } \\
\text { (líneas) }\end{array}$ \\
\hline \hline Jerpazsol & 2 \\
Los Libertadores & 5 \\
Tungurahua & 8 \\
Unión Ambateña & 5 \\
Vía Flores & 2 \\
\hline \hline
\end{tabular}

Fuente: Elaboración propia a partir de Dirección de Tránsito, Transporte y Movilidad DTM 2014

Para algunas preguntas de la encuesta, se calculó un Índice de percepción ciudadana, que toma valores de 0 a 100 puntos y cuyo cálculo consiste en la suma ponderada de las cuatro opciones de respuesta de cada pregunta.

Hay que tomar en cuenta que la percepción es un proceso subjetivo, selectivo y temporal, porque inciden intereses y actitudes personales, la experiencia, expectativas y las demandas de tipo temporal, situacional o social (Sánchez, R.,2014.49).

\section{Resultados}

\section{Aspectos generales}

En la investigación realizada el $60,5 \%$ de encuestados fueron mujeres y el $39,5 \%$ hombres, además los grupos de edad predominante son de $19-28$ años con $28,6 \%$, seguido del rango $29-38$ años con $23,6 \%$. El tiempo promedio de residencia en Ambato de los usuarios de buses es de 27 años con niveles de educación: bachillerato 43,8\%, superior $32,2 \%$ y educación básica $22,8 \%$. Información que se resume en las figuras 1 y 2 .

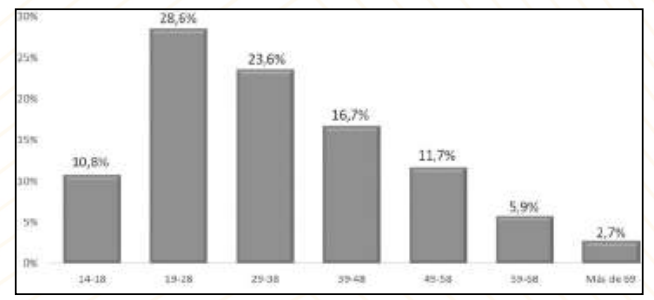

Fuente: Elaboración propia a partir de Encuesta de opinión febrero/2018 Figura 1.Edad de usuarios de buses urbanos cantón Ambato 2018

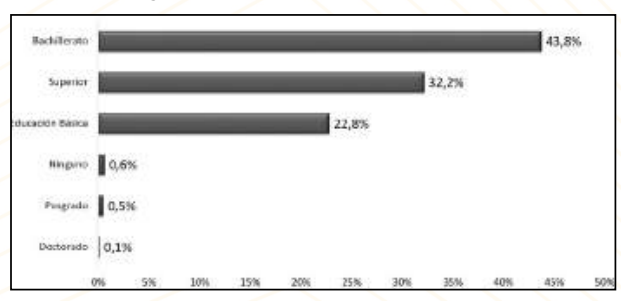

Fuente: Elaboración propia a partir de Encuesta de opinión febrero/2018 Figura 2. Nivel de educación de usuarios de buses urbanos cantón Ambato 2018

El $72,6 \%$ de los encuestados utilizan este medio de transporte diariamente y gastan un promedio de 1,20 USD, es decir, 36 USD mensuales, que representa el 9,33\% del Salario Básico Unificado-SBU 2018 (386 USD), cabe resaltar que, el ingreso promedio mensual de los encuestados es de 441,88 USD, donde el gasto alcanza el 8,14\%, con un sueldo máximo de 3.000 USD y un mínimo de 15 USD.

El principal motivo de viaje en bus es el traslado al trabajo $(41,6 \%)$ según la figura 3; y el 47,6\% toma más de un bus para llegar a su destino. Existe un 23,9\% de personas, quienes a pesar de tener vehículo propio utilizan bus, debido principalmente a que es usado por otro familiar (46,4\%), tráfico vehicular y dificultad para conseguir parqueo que juntas alcanzan el $36 \%$ como consta en la figura 4 . Estos problemas causan insatisfacción en los ciudadanos y conllevan al desorden de la ciudad, por ejemplo, el ayuntamiento de Madrid (2017), afirma que el tráfico se encuentra dentro de los tres principales problemas que afectan la calidad de vida y satisfacción con los servicios públicos de la ciudad.

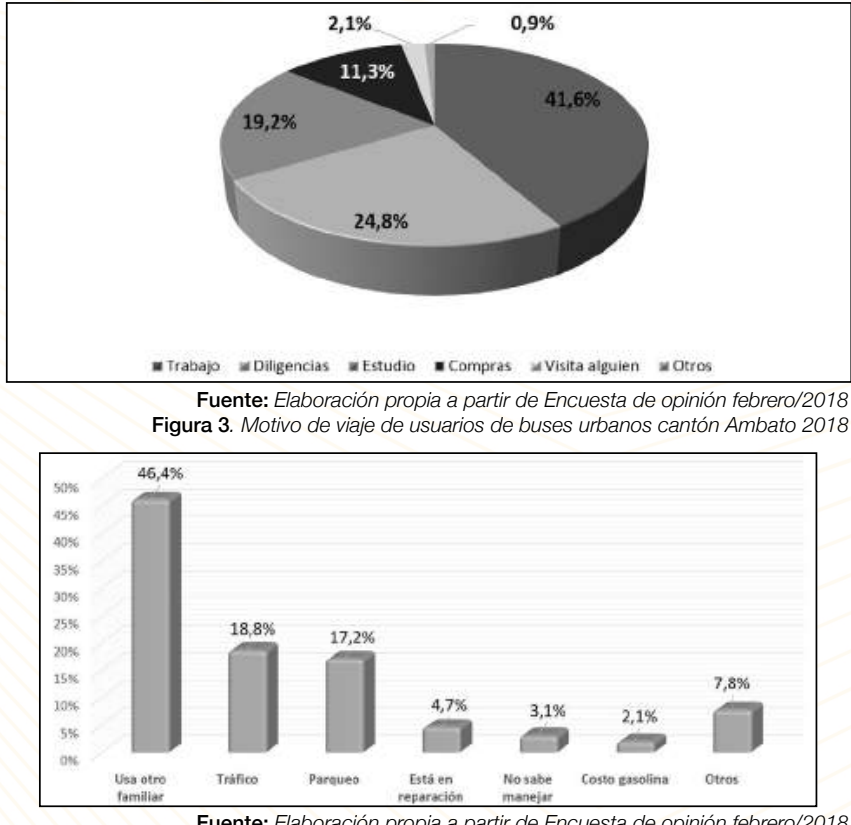

Figura 4. Motivos de utilización de buses por personas que poseen vehículo propio 2018

\section{Aspectos específicos}

\section{Infraestructura de unidades}

La mayoría de los usuarios han percibido una mejora en la infraestructura de las unidades, por lo que el 63,1\% piensa que el estado de los buses es bueno, el $25,8 \%$ regular, el $7,4 \%$ muy bueno y únicamente el 3,7\% indica que es malo, obteniendo un índice de 57,5 puntos, por otro lado se consultó acerca de la accesibilidad (facilidades para subir y bajar del bus), 61,1\% indica que es buena, $26,8 \%$ que es regular, $7,5 \%$ la califica como mala y $4,6 \%$ que es muy buena, generando un índice de 53,8 puntos, cuando el máximo esperado a alcanzar es 100 puntos como se observa en la figura 5. La percepción obtenida aún está alejada de la meta óptima de satisfacción, similar escenario se muestra en el estudio realizado por Ipsos Chile (2015), en la ciudad de Arica, donde la infraestructura es una de las dimensiones desfavorables.

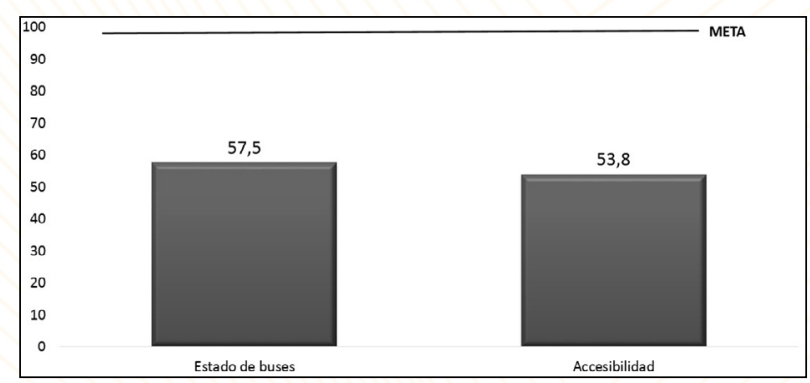

Fuente: Elaboración propia a partir de Encuesta de opinión febrero/2018 Nota: Los índices estado de buses y accesibilidad toma valores desde: $0=$ malo a $100=$ muy bueno Figura 5. Índices de percepción sobre infraestructura de buses urbanos cantón Ambato 2018

\section{Frecuencias, horario de servicio, tiempo y velocidad de buses}

El $55,4 \%$ de pasajeros afirma que la frecuencia con la que pasan los buses es buena, el 29,7\%regular, 8,1\% mala y el 6,9\% muy buena, registrando un índice de 53,2 puntos; en cuanto a los horarios de servicio el $64,5 \%$ está de acuerdo, el $24,8 \%$ en desacuerdo, el 6,1\% muy de acuerdo y 4,4\% muy desacuerdo, siendo su índice 56,8 puntos.

A pesar que Rivera et al (2017) indica que las frecuencias promedio de buses en el norte, centro y sur de Ambato son de 10,10 y 7,9 minutos respectivamente y el Plan Maestro de Transporte y Movilidad del cantón Ambato - PLAMTA (2013) menciona que el tiempo ideal de espera no debería ser mayor a 15 minutos, la ciudadanía percibe que aún se debe mejorar este aspecto.

Con respecto al tiempo que les toma a los usuarios llegar a su destino 
viajando en bus el $70,2 \%$ está de acuerdo, el 21,6\% en desacuerdo, el 5,1\% muy de acuerdo y $3,0 \%$ muy en desacuerdo, con un índice de 58,6 puntos.

La velocidad a la que viaja el bus es uno de los aspectos que los usuarios consideran importante, es así como el 52,9\% está de acuerdo, el 35,5\% está en desacuerdo, 7,6\% está muy desacuerdo y el 3,9\% muy de acuerdo teniendo un índice de 50,5 puntos. Cuatro de cada diez personas no están conformes con la velocidad pues indican que los choferes manejan a alta velocidad cuando están atrasados en su ruta y lento cuando la unidad tiene pocos pasajeros, además la velocidad afecta el momento de frenar para recoger y dejar pasajeros.

Información que se resumen en la figura 6.

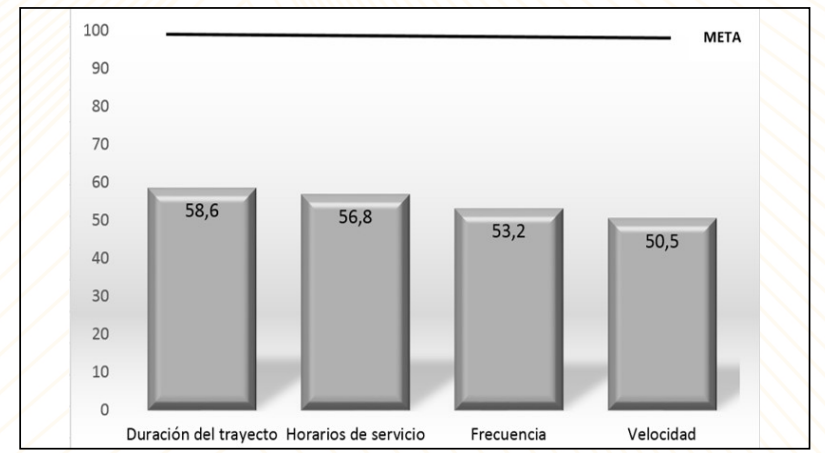

Fuente: Elaboración propia a partir de Encuesta de opinión febrero/2018 Nota: El índice de frecuencia toma valores desde: $0=$ malo a $100=$ muy bueno - Los índices duración del trayecto, horario de servicio y velocidad toman valores desde: $0=$ muy en desacuerdo a $100=$ muy de acuerdo

Figura 6. Índices de percepción sobre tiempo y velocidad de buses urbanos cantón Ambato 2018

\section{Paradas de buses}

Se consultó a los usuarios sobre la estructura de las paradas y el $53,5 \%$ considera que son buenas, el 32,8\% regulares, el 10,9\% malas y apenas el 2,9\% muy buenas, con estos porcentajes se calculó el índice de percepción, obteniendo como resultado 49,0 puntos. Además, se preguntó si la distancia entre paradas es adecuada, teniendo los siguientes resultados: el $78,6 \%$ están de acuerdo, el $15,8 \%$ en desacuerdo, el $3,7 \%$ muy de acuerdo y el $1,9 \%$ muy en desacuerdo, alcanzando un índice de 60,8 puntos.

Las paradas contribuyen a la calidad y funcionamiento ordenado del sistema de transporte de buses, incrementando la satisfacción del usuario. El índice obtenido, de 49 puntos, da a entender que este aspecto está un poco descuidado dentro del cantón, pues los encuestados mencionaron que las paradas del casco central de Ambato son las que se encuentran en mejor estado, mientras que, en las periferias hay déficit de infraestructura y señalización, como se evidencia en la figura 7. Como dato adicional el $58,7 \%$ de los encuestados utilizan las paradas autorizadas para subir o bajar de las unidades, lo cual indica en primera instancia que existe cultura vial por parte de los pasajeros, mientras que un $38,2 \%$ lo hace en paradas no autorizadas.

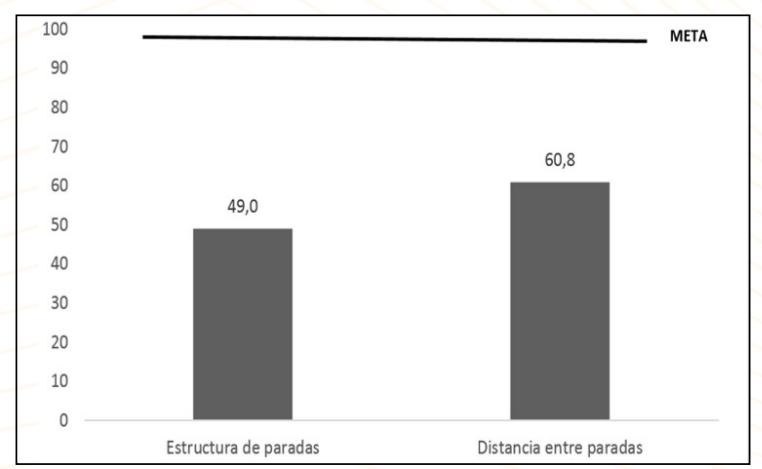

Fuente: Elaboración propia a partir de Encuesta de opinión febrero/2018 Nota: -El índice estructura de paradas toma valores desde: $0=$ malo a 100 = muy bueno - El índice distancia entre paradas toman valores desde: $0=m u y$ en desacuerdo a $100=$ muy de acuerdo

\section{Seguridad}

En lo referente a seguridad se formularon cuatro preguntas, que se muestran en la figura 8, las cuales evidencian que el $25 \%$ de encuestados ha sido víctima de algún acto delincuencial en las unidades, de los cuales el mayor porcentaje de víctimas se encuentran en el rango de edad de 19 - 28 años (29,6\%), seguido por el rango de $29-38(28,1 \%)$; sin embargo, el 54\%, cinco de cada diez pasajeros, conoce alguien que ha sufrido algún acto delincuencial mientras hacía uso de algún bus urbano; así mismo el 20\% mencionó haber sufrido algún accidente al subir o bajar del bus, siendo los de mayor frecuencia, las personas con edades comprendidas entre 19 y 38 años $(53,1 \%)$, lo que refleja una situación preocupante, pues el sistema de transporte público debe garantizar la seguridad, buen trato y respeto para los usuarios, un aspecto importante es que los choferes se detengan prudentemente y esperen el tiempo necesario para que los usuarios suban y bajen completamente en las paradas, tomando en consideración a los grupos de atención prioritaria (niñez, adultos mayores, mujeres embarazadas, personas con discapacidad).

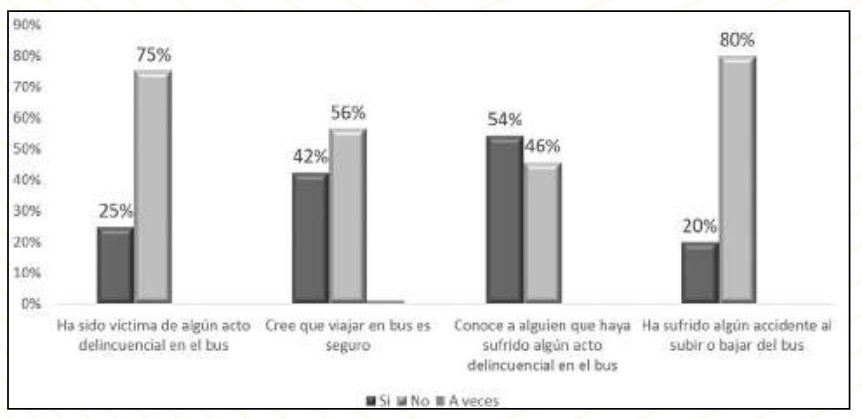

Fuente: Elaboración propia a partir de Encuesta de opinión febrero/2018 Figura 8. Aspectos sobre seguridad en buses urbanos cantón Ambato 2018

Un tema reciente que causa preocupación en los pasajeros es el incremento de vendedores ambulantes en los buses, pues el 40,9\% está en desacuerdo con su presencia, el 37,2\% muy en desacuerdo, 20,4\% de acuerdo y apenas el 1,0\% está muy de acuerdo, registrando un índice de 28,0 puntos. Si bien es cierto la situación económica del país genera incremento de comerciantes informales en las distintas ciudades, sin embargo, deberían buscarse soluciones de ubicación y orden por parte de las autoridades competentes, pues al analizar los datos numéricos de esta investigación más del $75 \%$ de usuarios de transporte público no está de acuerdo con la modalidad actual de trabajo.

\section{Comodidad}

Al analizar la comodidad física del usuario en el bus, el 73,8\% indicó que normalmente viaja sentado, lo cual guardaría relación con el 79,9\% que piensa que viajar en bus es cómodo, y a su vez esto contribuiría a que el $67,1 \%$ disfrute del viaje, según figura 9. Cabe indicar que la comodidad varía según el horario de uso del transporte, pues los encuestados manifiestan que en horas pico existe aglomeración de gente, aspecto que es mencionado de igual manera por Heredia (2015).

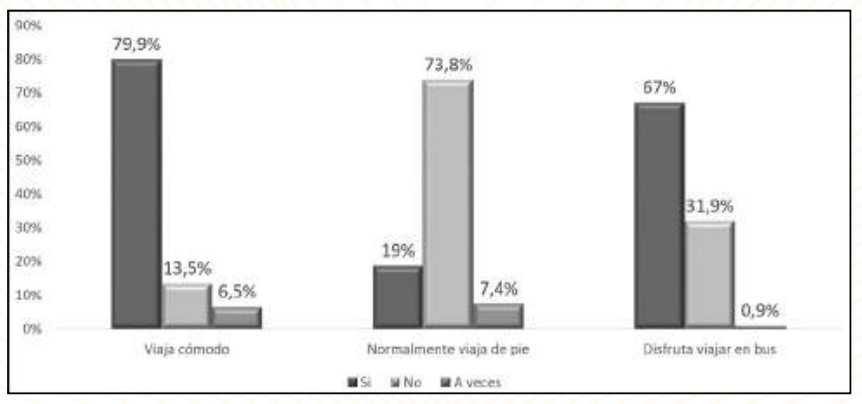

Fuente: Elaboración propia a partir de Encuesta de opinión febrero/2018 Figura 9. Aspectos sobre comodidad en buses urbanos cantón Ambato 2018

Figura 7. Índices de percepción sobre paradas de buses urbanos cantón Ambato 2018 


\section{Costo del pasaje y calidad de servicio}

El costo del pasaje general se incrementó de USD 0,25 a USD 0,30 y el pasaje de grupos de atención prioritaria de USD 0,10 a USD 0,15 a partir de mayo de 2015 (GADMA, 2015). Se consultó a los usuarios acerca de la aceptación de esta tarifa y se obtuvo que el 81,3\% está de acuerdo con el costo del pasaje, el 11,72\% está en desacuerdo, el $6,23 \%$ muy de acuerdo y $0,75 \%$ muy en desacuerdo, lo que da un índice de 63,8 puntos.

En Quito, el pasaje tiene un costo de USD 0,25, fijado en enero de 2003 y no ha sufrido modificación alguna en los últimos años debido a que el Municipio subsidia la tarifa invirtiendo alrededor de 20 millones de dólares anuales (El Comercio, 2017). En Ambato, los encuestados indicaron que el tiempo de vigencia de la tarifa de USD 0,30, ha influenciado para que la ciudadanía se acostumbre y esté de acuerdo con el valor establecido.

Tomando en cuenta los aspectos anteriores, los usuarios de los buses perciben en general que la calidad de servicio es buena (50\%), regular (34,9\%), mala $(10,1 \%)$ y muy buena (5\%), arrojando un índice de 49,5 puntos. A pesar de que la percepción de los pasajeros en más de la mitad de la muestra investigada es positiva, existen porcentajes relativos a deficiente calidad de servicio que deben ser tomados en cuenta para la propuesta de mejoras pertinentes, datos reflejados en la figura 10.

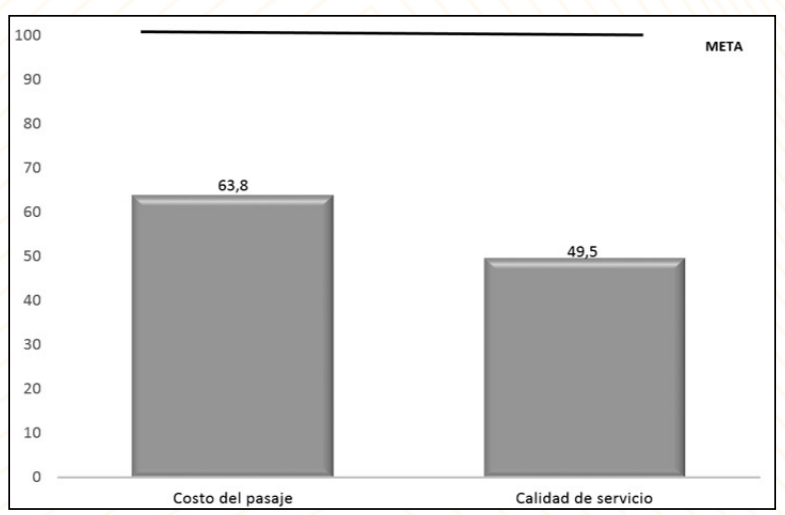

Fuente: Elaboración propia a partir de Encuesta de opinión febrero/2018 Nota:-El índice costo de pasaje toma valores desde: $0=$ muy en desacuerdo a 100= muy de acuerdo -El índice calidad de servicio toma valores desde: $0=$ malo a $100=$ muy bueno Figura 10. Índice de percepción sobre costo del pasaje y calidad del servicio

\section{Conclusiones}

El servicio de buses es utilizado con una frecuencia diaria por más del $70 \%$ de encuestados, quienes en su mayoría se movilizan al trabajo y gastan mensualmente el 9,33\% del SBU (2018), además una quinta parte de los usuarios poseen vehículo propio y se movilizan en bus debido principalmente a que es usado por otro familiar, tráfico intenso $\mathrm{y}$ falta de parqueo.

Más de la mitad de encuestados coinciden en que los buses se encuentran en buen estado, es decir se ha notado una mejora en su infraestructura. Sin embargo, el índice de percepción es de 57,5 puntos debido a que existe un $29,6 \%$ de personas que lo consideran entre regular y malo, entre las sugerencias recolectadas se indica que todavía existen unidades deterioradas y antiguas, que presentan deficiencias como el mal funcionamiento de puertas automáticas y exceso de smog que provoca contaminación del medio ambiente.

Los horarios de servicio y frecuencia del transporte urbano en la ciudad tienen índices de percepción de 56,8 y 53,2, respectivamente, lo que indica que existe un porcentaje considerable de usuarios que consideran aceptables estos aspectos, pero deben seguir mejorando pues los valores de los índices aún están muy alejados de la meta (100 puntos). Entre las sugerencias obtenidas de los encuestados está establecer la jornada completa de trabajo y mayor frecuencia los fines de semana, principalmente en las líneas: tres (Península-Centro- Las Orquídeas), siete (Letamendi, Atocha, Mirador) y once (Pucarumi, Cunchibamba, Tiugua).

El tiempo que toma viajar en bus no presenta mucha inconformidad por parte de los usuarios, posiblemente se deba a la extensión del cantón Ambato, lo que se refleja en su índice cercano a los 60 puntos; a pesar de ello, los usuarios muestran desacuerdo con la velocidad a la que transitan los buses, pues su índice es de 50,5 puntos, además, los encuestados sugieren que los choferes manejen con precaución y que se realice un mayor control de la velocidad.

La estructura de las paradas de bus es otra variable que presenta un índice bajo (49 puntos), entre las observaciones realizadas por los usuarios se menciona la insuficiencia de paradas y escasa señalización principalmente en sectores ubicados en las periferias de la ciudad, además, de que no reciben mantenimiento oportuno.

Si bien una cuarta parte de los encuestados no ha sufrido ningún acto delincuencial en el bus, el 54\% aseguró conocer a alguien que si lo sufrió; además, se menciona la intranquilidad por el aumento de vendedores dentro de las unidades, estas razones han contribuido para que el $56 \%$ de las personas entrevistadas perciban que viajar en bus no es seguro. Una de las sugerencias mencionadas por los usuarios para contrarrestar la inseguridad es que se aumente el número de cámaras en las unidades y se incremente el control a los vendedores.

Cerca del $70 \%$ de encuestados disfrutan viajar en bus ya que la mayoría viaja sentada, lo que contribuye a su comodidad, no obstante, los usuarios sugieren mejorar la calidad de los asientos.

El costo del pasaje en Ambato tiene aceptación de gran parte de los encuestados, su índice de percepción es de 63,8 puntos, empero, existen pasajeros que están inconformes con la tarifa comparada con la calidad de servicio que reciben, el cual tiene un índice de percepción de 49,5 puntos. Los usuarios sugieren que para mejorar el servicio se deben impartir cursos de capacitación de relaciones humanas a los choferes y controladores, no permitir llevar exceso de pasajeros, respetar las paradas, esperar a que los pasajeros suban y bajen de las unidades e incrementar controladores.

\section{Referencias}

Asociación León \& Godoy - Hidroplan. (2013). Informe Final: Detalle del Plan de Tránsito, Transporte y Movilidad del cantón Ambato. Quito.

Ayuntamiento de Madrid. (2017). Encuesta de Calidad de Vida y Satisfacción con los Servicios Públicos de la Ciudad de Madrid 2017. Recuperado de: http://www.madrid.es/portales/munimadrid/es/Inicio/El-Ayuntamiento/ Observatorio-de-la-Ciudad/Percepcion-Ciudadana/Edicion-2017?vgnextfmt=default\&vgnextoid=ba643225968b1610VgnVCM2000001f4a900aRCRD\&vgnextchannel=f22ff49c4495d310VgnVCM2000000c205a0aRCRD

Ambato, cada vez más cerca de la caja común en el servicio de transporte. (19 de agosto de 2017). El Telégrafo. Recuperado de: https://www.eltelegrafo.com.ec/noticias/regional-centro/1/ambato-cada-vez-mas-cerca-de-la-caja-comun-en-el-servicio-de-transporte

Banco de Desarrollo de América Latina - CAF. (2011). Desarrollo urbano y movilidad en América Latina. Recuperado de: https://www.caf.com/media/4203/desarrollourbano_y_movilidad_americalatina.pdf

Gobierno Autónomo Descentralizado Municipalidad de Ambato. (2015). Resolución de Concejo 233-2015. Recuperado de: http://gadmatic.ambato.gob. ec/lotaip/2015/Julio/anexo\%20literal\%20s/RC-233-2015.pdf

Heredia, J. (2015). Modelo de satisfacción de los usuarios de transporte público tipo bus integrando variables latentes. (tesis de posgrado). Universidad Nacional de Colombia. Medellín, Colombia

Instituto Ecuatoriano de Estadística y Censos - INEC. (2016). Estadísticas de transporte 2016. Recuperado de: http://www.ecuadorencifras.gob.ec/ transporte/ 
Instituto Ecuatoriano de Estadística y Censos - INEC. (2018). Proyecciones poblacionales. Recuperado de: http://www.ecuadorencifras.gob.ec/ proyecciones-poblacionales/

Ipsos. (2015). Medición y análisis de variables de operación y percepción usuaria de servicios de transporte público mayor urbano de Arica. Recuperado de http://www.dtpr.gob.cl/pdf/MenuSuperior/Estudios/XVNF\%20-\%20 INFORME\%2OFINAL\%20ESTUDIO\%20SATISFACCI\%C3\%93N\%20E\%2OIMAGEN\%2OTRANSPORTE\%2OPUBLICO\%2OARICA.pdf

Oviedo, N.V., Sacanambuy, J.M., Matabanchoy, S.M., y Zambrano C.A. (2016). Percepción de conductores de transporte urbano, sobre calidad de vida laboral. Universidad y Salud. 18(3), 432-446. DOI: http://dx.doi.org/10.22267/ rus. 161803.49

Pacheco Mayra. (29 de mayo de 2017). El subsidio al transporte público en Quito está en estudio. El Comercio. Recuperado de: http://www.elcomercio. com/actualidad/subsidio-transportepublico-quito-estudio-pasajes.html

Rivera, P., Mayorga, F., Vayas, T., Freire, L. (2017). El sistema de transporte público en el cantón Ambato. Frecuencias, productividad y velocidad. Boletín de Coyuntura. (13). 07-10.

\section{ESIC Editorial.}

Sánchez, R. (2014). Gestión y Psicología en empresas y organizaciones,

Sociedad Chilena de Ingeniería de Transporte- Sochitran. (2013). Desafíos del sector transporte. Urbano, 16 (28), 45-49. Recuperado de: http://www. redalyc.org/articulo.oa?id=19836965007

Vasconcellos, E. A., y Mendonça, A. (2016). Observatorio de Movilidad Urbana: Informe 2015-2016. Caracas.

Anexos página 25 